\title{
SOIL MOISTURE REMOTE SENSING FOR NUMERICAL WEATHER PREDICTION: L-BAND AND C-BAND EMISSION MODELING OVER LAND SURFACES, THE COMMUNITY MICROWAVE EMISSION MODEL (CMEM)
}

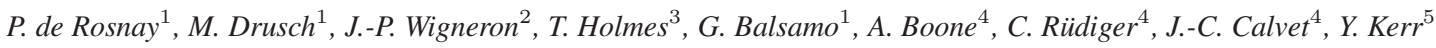

(1) European Centre for Medium Range Weather Forecasts, Shinfield Park, RG2 9AX, Reading, UK

(2) INRA/EPHYSE, Bordeaux, France, (3) Vrije Universiteit Amsterdam, The NetherLand

(4) Météo-France/CNRM, Toulouse, France, (5) CESBIO, Toulouse, France.

\begin{abstract}
The Community Microwave Emission Model (CMEM) is the low frequency forward observation operator developed at ECMWF. It is used in this paper to simulate brightness temperatures at local and regional scales over SMOSREX (France) and AMMA (West Africa), respectively. Background errors in simulated brightness temperatures are quantified at different frequencies and incidence angles for these two sites.
\end{abstract}

Index Terms - CMEM, SMOSREX, AMSR-E, ALMIP, SMOS

\section{INTRODUCTION}

The Soil Moisture and Ocean Salinity (SMOS) satellite, to be launched in early 2009, will be the first mission to provide global fields of L-Band Brightness Temperature [1]. SMOS brightness temperatures will be used at the European Centre for Medium-Range Weather Forecasts (ECMWF) to analyze soil moisture through the Surface Data Assimilation System (SDAS). This is expected to improve the accuracy of initial conditions of the Numerical Weather Prediction (NWP) model. The Community Microwave Emission Model (CMEM) has been developed at ECMWF for forward modeling of passive microwave emission of the surface at low frequency (1 GHz to $20 \mathrm{GHz}$ ) [2, 3]. CMEM is a highly modular microwave emission model that allows considering different parameterizations for each component of the soil-vegetation-atmosphere dielectric layers.

In this paper CMEM is shortly described. Background errors in simulated brightness temperature are quantified at different spatial scale, based on the Surface Monitoring Of the Soil Reservoir EXperiment (SMOSREX) and the African Monsoon Multidisciplinary Analysis (AMMA) experiment.

\section{THE COMMUNITY MICROWAVE EMISSION MODEL}

The Community Microwave Emission Model has been developed by the European Centre for Medium-Range Weather Forecasts (ECMWF) as the forward operator for low frequency passive microwave brightness temperatures (from $1 \mathrm{GHz}$ to $20 \mathrm{GHz}$ ) of the surface $([2,3])$. It is a new highly modular software package providing Input/Output (I/O) interfaces for the Numerical Weather Prediction Community. CMEM's physics is based on the parameterizations used in the L-Band Microwave Emission of the Biosphere (L-MEB, [4]) and the Land Surface Microwave Emission Model (LSMEM, [5]). CMEM modularity allows considering different parameterizations of the soil dielectric constant as well as different soil approaches (either coherent or incoherent) and different effective temperature, roughness, vegetation and atmospheric contribution models (Table 1). According to the number of options provided in Table 1 CMEM allows considering 1440 different configurations to simulate Top Of Atmosphere brightness temperatures. CMEM is fully described under http://www.ecmwf.int/research/ ESA_projects/SMOS/cmem/cmem_index.html.

A convergence study has been performed at L-band and at the tile scale between the CMEM and the LMEB models. To this end CMEM has been used in the LMEB configuration as indicated by the bold options in Table 1. CMEM and LMEB simulated brightness temperatures are compared for nine types of vegetation (bare soil, water, c3 grass, c4 grass, c3 crops, c4 crops, deciduous forest, coniferous forest, tropical forest). Root mean square difference between the models is very small (in the range of $0.01 \mathrm{~K}$ to $0.001 \mathrm{~K}$ ) at any incidence angle for any vegetation type. The residual difference, still larger that computer accuracy, is due to input parameters and physical constants that are not truncated at the same number of decimal in the two models. This convergence investigation ensures that, when appropriate set of options is chosen, CMEM is in agreement with LMEB and the SMOS retrieval algorithm.

In this paper, the modularity of CMEM is used to address the sensitivity of the simulated brightness temperature to different configurations concerning the vegetation optical depth modeling and the soil dielectric constant.

\section{BACKGROUND ERRORS OVER SMOSREX}

The Surface Monitoring Of the Soil Reservoir EXperiment (SMOSREX) provides a continuous 2003-2008 complete ground and remotely sensed data set at local scale [22]. Soil moisture and temperature profiles, meteorological variables and multi-spectral measurements, including high accuracy L-Band, have been continuously recorded at time steps ranging from $2 \mathrm{~min}$ to $30 \mathrm{~min}$. Vegetation characteristics have also been monitored on a monthly basis. In the present study, the 2004 data set is used to investigate CMEM potential for future SMOS data assimilation. Background errors of the ECMWF simulated L-band brightness temperature are investigated over SMOSREX. The operational NWP atmospheric conditions from ECMWF are used to run the HTESSEL Land Surface Model (LSM), coupled to CMEM at the T799 spatial resolution $\left(0.225^{\circ}\right)$. Model-based L-band brightness temperatures are evaluated against the SMOSREX data set, for temporally and spatially collocated data. This comparison is based on the use of four statistical indices: bias, RMSE (root mean square error), determination coefficient $\left(R^{2}\right)$, and efficiency (Nash coefficient). Figure 1 illustrates dif- 
Table 1. Physical parameterizations used in CMEM for the soil, vegetation and atmospheric dielectric layers. CMEM is equivalent to LMEB ([4]) when options in bold are chosen.

\begin{tabular}{l|c} 
Model & Parameterizations \\
\hline Soil dielectric constant & Dobson / Mironov / Wang \& Schmugge $[6,7,8]$ \\
Effective Temperature & Choudhurry / Holmes / Wigneron [9, 10, 11] \\
Smooth emissivity & Fresnel law / Wilheit [12] \\
Rough emissivity & ATBD / Choudhurry / Wegmüller / Wigneron01 / Wigneron07 [13, 14, 15, 11, 4] \\
\hline Vegetation Opacity & Jackson, Kirdyashev, Wegmüller, Wigneron $[16,17,18,4]$ \\
\hline Atmosphere Opacity & Liebe, Pellarin, Ulaby [19, 20, 21] \\
\hline
\end{tabular}
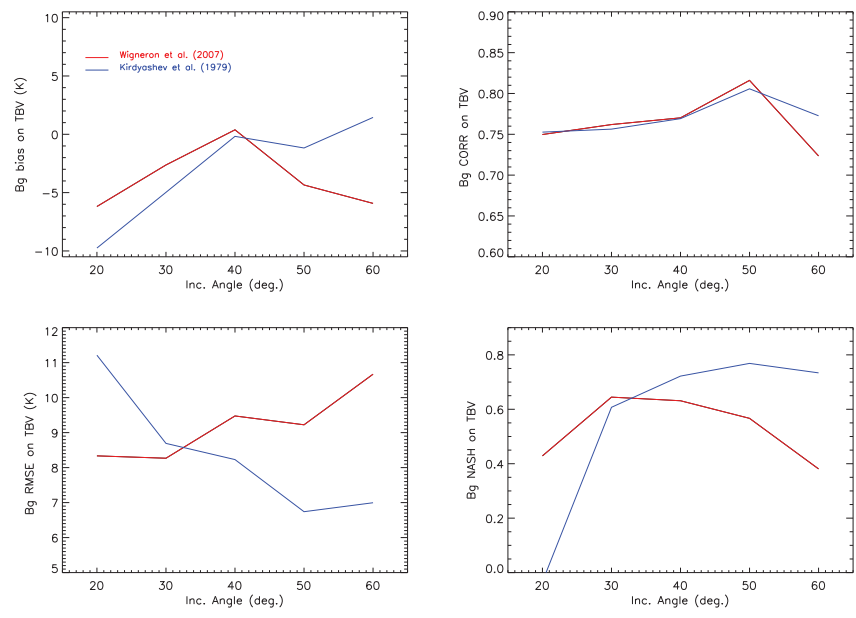

Fig. 1. Background error of the ECMWF synthetic brightness temperature $(\mathrm{K})$ over the SMOSREX pixel, as a function of the incidence angle, at vertical polarization for two different microwave modeling approaches of the vegetation optical depth by [4] and [17] (Table 1).

ferences between modeled and observed brightness temperature at vertical polarization for two different modeling configurations of the vegetation optical depth (Wigneron and Kirdyashev parameterizations) as a function of incidence angle. For both the Wang and Schmugge dielectric model is used since it provides better agreement with the SMOSREX observations compared to the other dielectric models of Table 1 . The background error in simulated brightness temperature is sensitive to the observing incidence angle. When considering Wigneron's parameterization of vegetation optical depth the minimum bias is obtained for an incidence angle of $40^{\circ}$. The determination coefficient $\left(R^{2}\right.$, square correlation) between modeled and observed brightness temperature for the whole annual cycle, indicates that the temporal dynamics are very well captured by the simulated brightness temperatures at any incidence angle, although best correlation $(\mathrm{R} 2=0.82)$ is obtained at $50^{\circ}$. RMSE in simulated brightness temperature increases with the incidence angle and the Nash coefficient indicates best performances of the synthetic ECMWF's brightness temperatures for observing angle of $30^{\circ}$ when the Wigneron's parameterization is used in the forward model to account for the vegetation optical depth.

When the Kirdyashev model is used in the forward operator, best performances are obtained for incidence angles between $40^{\circ}$ and $60^{\circ}$ than for lower angles. The overall best modeling/observing configuration is obtained when the Kirdyashev opacity model is used for observing angle of $50^{\circ}$. This result provides a preliminary indication of an optimal observing/modeling configuration. It is also suggested that the incidence angle should be used in the bias correction scheme. The good agreement obtained in terms of determination coefficient (higher than 0.7 in any conditions) between modeled and observed brightness temperature at vertical polarization is particularly noteworthy. It provides an indirect validation of the ECMWF's operational model over this site. It indicates that simulated atmospheric, soil moisture and soil temperature conditions are consistent with those observed over SMOSREX.

\section{EVALUATION OF CMEM BRIGHTNESS TEMPERATURE OVER WEST AFRICA: ALMIP-MEM}

A larger scale evaluation of CMEM is presented over West Africa in the joint context of the AMMA Land Surface Models Intercomparison Experiment (ALMIP, http://www.cnrm.meteo.fr/ammamoana/amma_surf/almip/index.html) [23] and the future SMOS validation over West Africa ([24, 25]). Simulated brightness temperatures (TB) by different ALMIP land surface models-CMEM are evaluated against AMSR-E C-band data for 2006. Figure 2 represents the time-latitude diagram of the horizontally polarized brightness temperatures at C-band for AMSR-E and simulated. For each LSM a simple bias correction has been taken into account to correct the simulated brightness temperatures based on the annual mean bias value. The time-latitude diagram shows the simulated brightness temperature when CMEM is used with the Kirdyashev vegetation opacity model and the Wang and Schmugge dielectric model. AMSR-E C-band data show a wet patch over Sahel during the rainy season, centered at Day of year 210 and latitude $15.5^{\circ}$ North. This wet patch is captured by all the LSMs, but the amplitude is either overestimated or underestimated depending on the LSM. However this figure underlines the general good agreement between the forward approach and the satellite data.

A condensed quantitative view of the ALMIP-MEM results is provided by Figure 3 which shows Taylor diagrams between simulated and observed C-band brightness temperatures. Taylor diagrams display the standard deviation (SDV) as a radial distance and the correlation with observations as an angle in the polar plot. They also indicate the centered RMS difference between the simulated and observed patterns, as the distance to the point marked "Data" on the x-axis. SDV are normalized by the SDV of the AMSR-E observations. The Taylor diagrams summarize here the relative skill with which the different models simulate the spatial and temporal pattern of $\mathrm{C}$-band brightness temperatures. Best simulations are the nearest to the point "Data", indicating they have high correlation and low RMS difference with observations. Models lying on the dashed arc have the correct standard deviation, which indicates that 

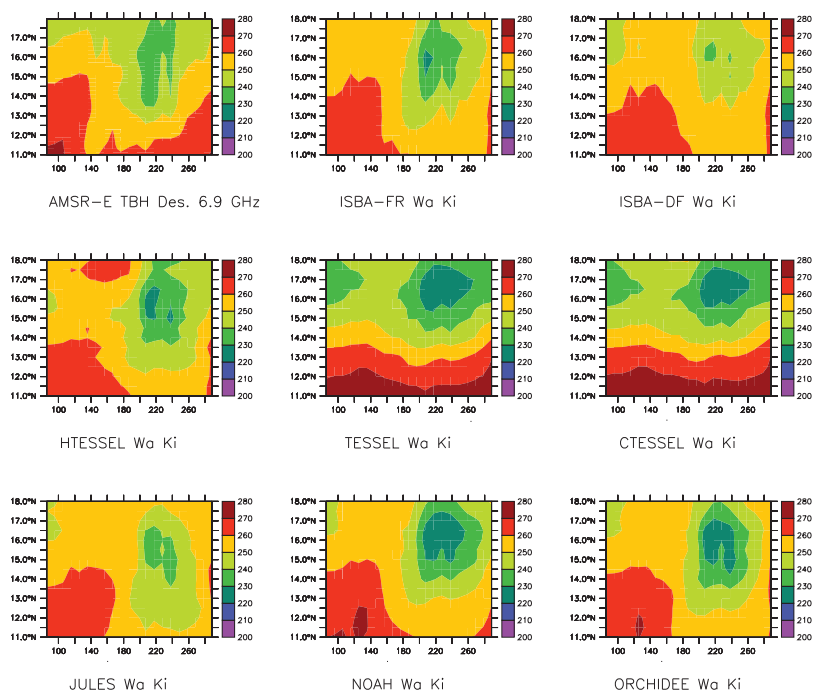

Fig. 2. Time-latitude diagram of the horizontally polarized brightness temperature $(\mathrm{K})$ observed by AMSR-E and simulated by ALMIP-MEM. Time axis is in Day of Year. For each ALMIP-MEM simulation a bias correction was applied, specifically computed for each LSM when comparing simulated and observed brightness temperature.

the amplitude of simulated variations are in agreement with that of observations. Figure 3 (a) is the Taylor diagram obtained for different LSMs using the best microwave modeling configuration (Wang and Schmugge for dielectric model and Kirdyashev for vegetation opacity). Each LSM uses the same atmospheric forcing and the same microwave emission model. The scatter between the LSMs is due to differences in land surface processes modeling (different simulations of soil moisture and soil temperature profiles). Figure 3 (b) shows HTESSEL results for the 12 possible different cross configurations of microwave emission modeling considered for dielectric and vegetation opacity models in CMEM (Table 1). Best models in Figure 3 (b) are shown by indexes 2, 6, 10, all corresponding to the Kirdyashev opacity model with the Dobson, Mironov and Wang and Schmugge dielectric model, respectively. Relative SDV is in the range of 0.67 to 1.36 and correlation values between modeled and observed brightness temperatures vary between 0.54 and 0.73 depending of the considered LSM. The scatter in model performances lies in the rang of 1.01 to 1.66 and from -0.16 to 0.54 for the correlation. In these simulations, soil moisture and soil temperature profiles are identical, but the parameterization of the soil dielectric constant and vegetation opacity are different. Comparison of Figure 3 (a) and (b) clearly shows that the scatter due to the microwave emission model is much larger than that due to the LSMs. This figure also points out that the Kirdyashev model leads to much better performances than the other vegetation opacity models, in terms of both RMSE and correlation coefficient. The Wang and Schmugge model provides best results, whatever the opacity model used for the vegetation.
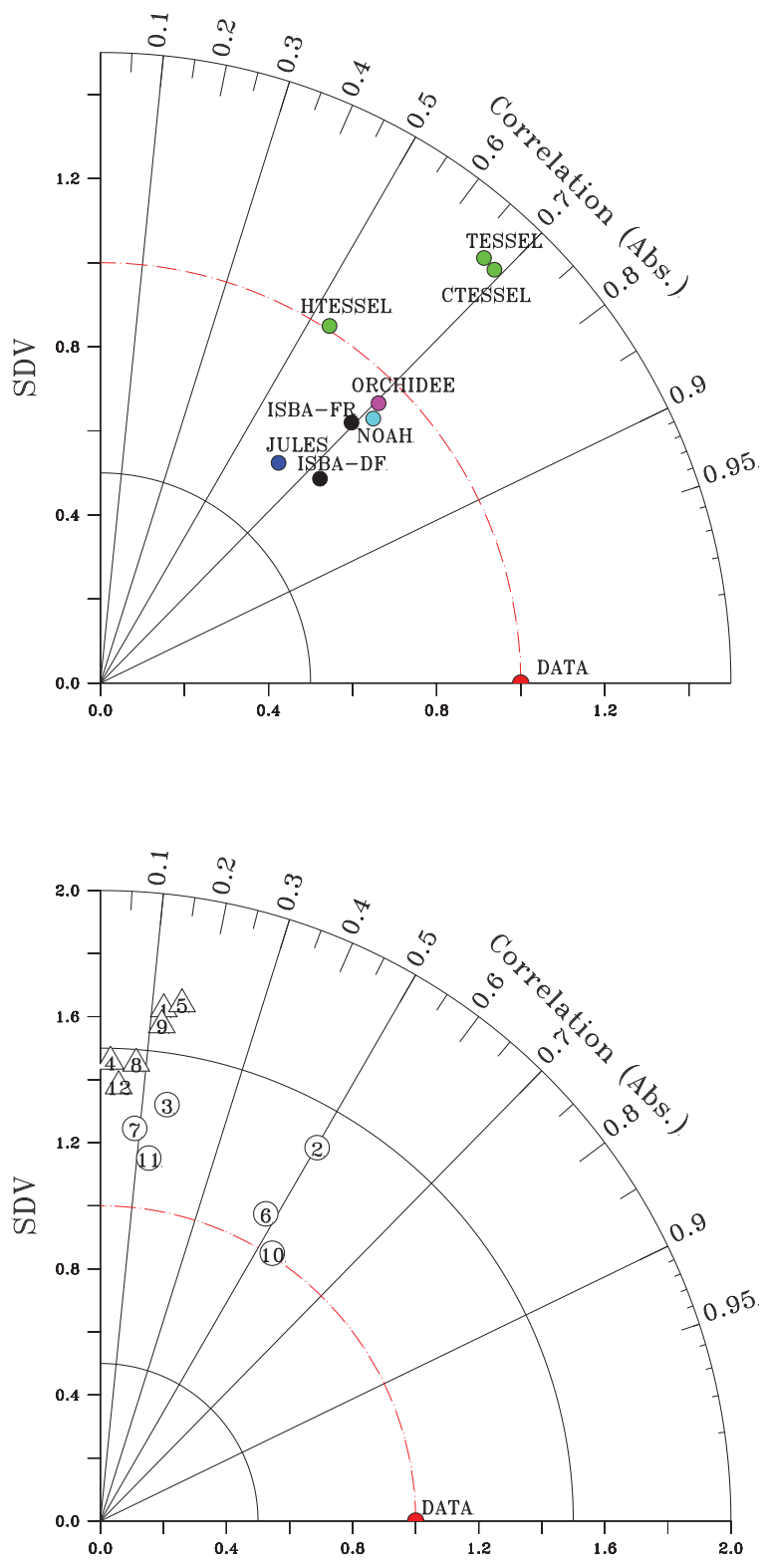

Fig. 3. Taylor diagrams illustrating the statistics of the comparison between ALMIP-MEM synthetic brightness temperature and AMSR-E data at C-band for (a) different LSMs coupled to CMEM using the Wang and Schmugge dielectric model coupled to the Kirdyashev vegetation opacity model, (b) the HTESSEL LSM coupled to CMEM using 12 different configurations of the microwave emission modeling for vegetation and soil dielectric constant (Table 1). Circles indicate positive correlation values and triangles indicate negative correlations. Note that the radial axis scale is different for (a) and (b). 


\section{CONCLUSION}

CMEM is used in this paper to simulate brightness temperatures at local scale and regional scale over the SMOSREX and AMMA sites. Background errors in simulated brightness temperature are quantified at L-band in multi-angular configuration over SMOSREX using LEWIS field measurements, and at C-band over West Africa using AMSR-E C-band satellite data. For both, the sensitivity of simulated brightness temperature to the choice of vegetation opacity model and dielectric model is investigated. On the two sites the Wang and Schmugge dielectric model provides best agreement with observed brightness temperatures. For SMOSREX the Wigneron and the Kirdyashev opacity models perform well to reproduce temporal dynamics of brightness temperature at L-band at low and high incidence angle respectively. Over West Africa, validation against C-band AMSR-E data indicates that the Kirdyashev opacity model is best suited to reproduce spatial and temporal pattern of brightness temperature at this frequency. These results obtained at different frequencies, different spatial resolution and for several incidence angles, point out the importance of the forward modeling approach for the accuracy of the simulated brightness temperature over land. They allow identifying the best suitable microwave emission modeling approach for the future SMOS monitoring and for the assimilation of brightness temperature over land surfaces.

\section{REFERENCES}

[1] Kerr, Y. H. et al., "Soil moisture retrieval from space: The soil moisture and ocean salinity (smos) mission," IEEE Trans. Geosc. Remote Sens., vol. 39 (8), pp. 1729-1735, 2001.

[2] Drusch, M. et al., "Comparing ERA-40 based L-band brightness temperatures with Skylab observations: A calibration / validation study using the Community Microwave Emission Model," J. Hydrometeo, vol. under revision, 2008.

[3] Holmes, T. et al., "A global simulation of microwave emission: Error structures based on output from ECMWFs operational Integrated Forecast System," IEEE Trans. Geosc. Remote Sens., vol. 46(3), pp. 846-856, 2008.

[4] Wigneron, J.-P. et al., "L-band Microwave Emission of the Biosphere (L-MEB) Model: description and calibration against experimental data sets over crop fields.," Remote sens. environ., vol. 107, pp. 639-655, 2007.

[5] Drusch, M. et al., "Vegetative and atmospheric corrections for soil moisture retrieval from passive microwave remote sensing data: Results from the Southern Great Plains Hydrology Experiment 1997," J. Hydrometeo, vol. 2, pp. 181-192, 2001.

[6] Dobson, M.C. et al., "Microwave dielectric behavior of wet soil-partii: Dielectric mixing models," IEEE Trans Geosc. Sci, vol. 38, pp. 1635-1643, 1985.

[7] Mironov, V.L. et al., "Generalized refractive Mixing dielectric model for moist soils," IEEE Trans. Geosc. Remote Sens., vol. 42(4), pp. 773-785, 2004.

[8] Wang, J. R. and Schmugge, T., "An empirical model for the complex dielectric permittivity of soils as a function of water content," IEEE Trans. Geosc. Remote Sens., vol. 18, pp. 288295, 1980.

[9] Choudhury, B.J. et al., "A parameterization of effective soil temperature for microwave emission," J. Geophys. Res., pp. 1301-1304, 1982.
[10] Holmes, T. et al., "A new parameterization of the Effective Temperature for L-band Radiometry," Geophy. Res. Letters, vol. 33, pp. L07405, doi:10.1029/2006GL025724, 2006.

[11] Wigneron, J.-P. et al., "A Simple Parmeterization of the L-band Microwave Emission from Rough Agricultural Soils," IEEE Trans. Geosc. Remote Sens., vol. 39, pp. 1697-1707, 2001.

[12] Wilheit, T. T., "Radiative transfert in plane stratified dielectric," IEEE Transactions on Geoscience Electronics, vol. 16(2), pp. 138-143, 1978.

[13] SMOS. ATBD, "SMOS level 2 Processor for Soil Moisture Algorithm Theoretical Based Document (ATBD)," SO-TN-ESLSM-GS-0001, issue 2.a, 2007.

[14] Choudhury, B.J. et al., "Effect of surface roughness on the microwave emission from soils," J. Geophys. Res., pp. 56995706, 1979.

[15] Wegmüller, U. and Mätzler, C., "Rough bare soil reflectivity model," IEEE Transactions on Geoscience Electronics, vol. 37, pp. 1391-1395, 1999.

[16] Jackson, T.J. and Neill, O., "Attenuation of soil microwave emission by corn and soybeans at 1.4and 5 ghz," IEEE Trans. Geosc. Remote Sens., vol. 28(5), pp. 978-980, 1990.

[17] Kirdyashev, K.P. et al., "Microwave radiation of the earths surface in the presence of vegetation cover," Radiotekhnika $i$ Elektronika, vol. 24, pp. 256-264, 1979.

[18] Wegmüller, U. et al., "Canopy opacity models, in passive microwave remote sensing of land-atmosphere interactions," $B$. et al. Ed. Utrecht, The Netherlands: VSP, p. 375, 1995.

[19] Liebe, H.J., "MPM- An atmospheric millimeter-wave propagation model," Int. J. Infrared Millimeter Waves, vol. 10, pp. 631-650, 2004.

[20] Pellarin, T. et al., "Two-year global simulation of L-band brightness temperature over land," IEEE Trans. Geosc. Remote Sens., vol. 41(4), pp. 2135-2139, 2003.

[21] Ulaby, F.T. et al., ," in Microwave remote sensing: active and passive, Vol III, from theory to application. 1986, Artech House, Dedham, MA.

[22] de Rosnay, P. et al., "SMOSREX: A long term field campaign experiment for soil moisture and land surface processes remote sensing," Remote sens. environ., vol. 102, pp. pp 377-389; doi:10.1016/j.rse.2006.02.021, 2006.

[23] A. Boone and P. de Rosnay, "Toward the improved understanding of land-surface processes and coupling with the atmosphere over West Africa," LEAPS Newsletter 3, 2007.

[24] de Rosnay, P. et al., "Multi-scale soil moisture measurements over the Gourma meso-scale site in Mali," J. hydrol. submitted, 2008.

[25] de Rosnay, P. et al., "Multiscale validation of SMOS brightness temperature and products over West Africa.," SMOS cal-val ESA ID 3257, pp. Available on http://www.cesbio.upstlse.fr/us/indexsmos.html, 2005. 